\title{
Metapopulation mean life time within complex networks
}

\author{
S. Kininmonth ${ }^{1,2, *}$, M. Drechsler ${ }^{3}$, K. Johst ${ }^{3}$, H. P. Possingham ${ }^{2}$ \\ ${ }^{1}$ Australian Institute of Marine Science, PMB 3, Townsville, Queensland 4810, Australia \\ ${ }^{2}$ The Ecology Centre and Commonwealth Research Facility for Applied Environmental Decision Analysis, School of Biology, \\ The University of Queensland, Brisbane, Queensland 4072, Australia \\ ${ }^{3}$ Department of Ecological Modelling, UFZ-Helmholtz Centre for Environmental Research, Permoserstr. 15, 04318 Leipzig, \\ Germany
}

\begin{abstract}
Metapopulation dynamics depend on the exchange of individuals between populations across the landscape. The environment that the migrants must traverse is influenced by many forces, so the connections are often complicated pathways, which can be represented as a network. The structure of these networks will determine which populations will receive more migrants than other populations, and this in turn affects the lifetime of the metapopulation. We present a modification of the Drechsler (2009) formulae for the mean lifetime of a metapopulation based on network properties where the arrangement of the population connections is not limited to simple dispersal kernels. We provide a graph-theoretical framework for analysing the dispersal network and apply this model to the small-world network of the Great Barrier Reef as well as to conservation planning in general. Our results highlight that the topology of the dispersal network strongly influences the metapopulation mean lifetime. Metapopulation models and conservation planning need to include the capacity for basing interactions on complex topological structures.
\end{abstract}

KEY WORDS: Metapopulation $\cdot$ Dispersal $\cdot$ Networks $\cdot$ Graph theory

\section{INTRODUCTION}

Fundamental to the persistence of populations in a heterogeneous environment is the capacity to exchange individuals between locations and thus compensate local extinction through colonisation (Hanski 1998). The exchange process is particularly evident in marine ecosystems where coral larval dispersal events create extensive planktonic slicks (Oliver \& Willis 1987). However, this massive exchange of coral and, to a lesser extent, fish larvae from one population to another is complex in structure and highly variable through time (James et al. 2002, Jones et al. 2005, Underwood et al. 2007, Graham et al. 2008, van Oppen et al. 2008). In contrast to simple dispersal kernels often used in metapopulation modelling, models that simulate dispersal of individuals as a network often demonstrate the local and regional complexity of metapopulation connectivity (Treml et al. 2008, Urban et al. 2009). Modelling metapopulations in such a complex environment remains a challenge, notwithstanding advances in the fields of hydrodynamics and metapopulation theory (Crowder et al. 2000, Bode et al. 2008). However, metapopulation models are necessary for evaluating and guiding conservation planning, especially in reserve system design and spatial prioritisation of complex seascapes (Gerber et al. 2003, Kritzer \& Sale 2004, Crowder \& Figueira 2006, Kaplan 2006, Nicholson \& Possingham 2007, Moilanen et al. 2009).

Metapopulation theory has been extensively developed since the early modelling of agricultural systems by Levins (1969). Early models with deterministic equations and fixed parameters have been slowly replaced by stochastic models with uncertain parameters (Hanski 1998, Ovaskainen et al. 2002, Wilcox \& Possingham 2002), but these are based on a simple 
dispersal framework. Dispersal is generally modelled as a distance function that distributes propagules in a radiating manner from the boundaries enclosing the habitat. The fundamental assumption here is that dispersal functions in a static homogeneous matrix whose spatial configuration solely determines dispersal success. In marine ecosystems, the dynamic nature of the ocean, driven by tides, wind, upwelling and oceanic currents, imposes a significant influence on dispersal distances and directions, especially for passive larvae (Hughes et al. 1999, Kingsford et al. 2002).

This marine metapopulation paradigm, despite an absence of recorded extinction events, takes a more modern approach as outlined by Sale et al. (2006). This approach, where the emphasis is on the dynamics of species in a patchy environment, is applicable for species that have adults that remain restricted to isolated physical structures such as coral reefs and rocky shores. The recent global bleaching events (Wilkinson 2000) have highlighted the need to consider populations as potentially isolated from colonisation processes (Underwood et al. 2009). However, metapopulation dynamics is not just a function of larval dispersal but includes demographic rates of survival, growth and reproduction (Crowder et al. 2000) specific to each habitat patch. In this paper we will refer to 'patch' as the discrete habitat utilised by a population. Habitat quality will determine if the habitat patch acts as a 'source' or 'sink' (Pulliam 1988) for the local populations. Habitat quality is commonly heterogeneous at a regional scale, and understanding the interplay between larval supply and habitat quality is crucial for conservation planning (Lipcius et al. 2001, 2008).

Frank \& Wissel $(1998,2002)$ modelled heterogeneous landscapes by explicitly including variation in both inter-patch distance and patch size. Drechsler (2009) adopted the Frank \& Wissel (2002) metapopulation mean life time (MLT) model and developed an analytical formula for metapopulation viability, which is based on macroscopic habitat network properties (the ratios of dispersal range and environmental correlation range to network geographic size, and the total number and [geometric mean] size of the patches). The aggregated framework provided by Drechsler (2009) is especially appropriate for application to graph theory. However, the larval dispersion considered by Drechsler (2009) was based on the Euclidean distance dispersal, and was thus limited to situations where the matrix is homogeneous and organisms show no directed movement. Applying a dynamic dispersal model, such as the Lagrangian particle model by James et al. (2002), can enable more complex dispersal networks. The dispersal model can be moved out of geometric space and be based within a graph theoretical framework (see Appendix 1).
The network approach has been used for metapopulation modelling (Bascompte \& Sole 1996, Ovaskainen \& Hanski 2001, Urban \& Keitt 2001, Franc 2004, Colizza et al. 2007, Bode et al. 2008, Rozenfeld et al. 2008). While these use networks to describe the complex interactions, we found the simplicity of Drechsler's (2009) equations the most adaptable to a graph theory approach. In particular, the macroscopic approach, using mean field methods based on patch scale dynamics, to evaluate the entire network was suitable for application to more complex dispersal structures.

The dispersal of coral and fish larvae throughout the Great Barrier Reef (GBR) in Australia can be represented as a complex network (Kininmonth et al. 2010). The larval processes of spawning, drifting, swimming, and settling combined with predation in a Lagrangian particle model (James et al. 2002) capture some of the complex interactions between coral reefs that are many $\mathrm{km}$ apart (Bode et al. 2006). The heterogeneous distribution of coral reef area, number of connections and the weight of connections can be included as integral components of the network.

The recent examination of naturally forming networks (Steuer \& Lopez 2008), from food webs (Montoya \& Sole 2002) to metabolic pathways (Jeong et al. 2000) to the regional dispersal patterns of coral and fish (Kininmonth et al. 2010), show a dominant pattern termed a 'small-world' (Grossman 2000). This topological configuration applies to networks where the average path length increases more slowly than or equally to the logarithm of network size (Junker \& Schreiber 2008). We use the term 'vertices' (=nodes) to describe the graph-theoretical feature (see Appendix 1) used to represent an entire patch. The small-world effect is that even with a large increase in network size, the distance from one vertex to any other remains small.

Based on the equations from Drechsler (2009), we develop a framework showing how graph theory, in terms of dispersal network structure (Kininmonth et al. 2010), can be integrated into metapopulation theory. Using this framework, we explore how network topology influences MLT of a metapopulation. Natural systems can interact using a wide array of topologies from random to lattice to small-world (see Table 1), and understanding how these arrangements affect metapopulation persistence is critical to conservation planning. We then apply the new model to the GBR larval dispersal network described by Kininmonth et al. (2010).

\section{METHODS}

Integration of network topology into metapopulation theory. The models of Drechsler (2009) contain 3 basic rates characterising the dynamics of metapopulations: 
(1) extinction rate of local populations on patch, $i$, as:

$$
V_{i k}=\varepsilon A_{i}^{-\eta}
$$

where $A_{i}$ is patch area, $\varepsilon$ is a species-specific $(k)$ coefficient relating to minimum patch size, and $\eta$ is a scaling parameter describing the level of environmental variation in the local population growth. A smaller value of $\eta$ indicates more environmental variability and consequently a relatively larger extinction rate (Eq. 1 in Drechsler 2009).

(2) Colonisation strength (Eq. 3 in Drechsler 2009) of patch $i$ describing its ability to colonise another patch:

$$
C_{i}^{\text {out }}=\frac{\beta A_{i}^{b}}{(N-1) \mu} \sum_{j(\neq i)} \exp \left(-\alpha d_{i j}\right)
$$

which is given by the emigration rate $n_{i}=\beta A_{i}^{b}$ of a patch $i$ where $\beta$ and $b$ are species-specific constants, the number of patches $N$, the number of immigrants required for successful colonization $\mu$, the distances $d_{i j}$ between patch $i$ and $j$, and the mean dispersal distance of a species $(1 / \alpha)$.

(3) Colonisability of patch $i$ (Eq. 4 in Drechsler 2009) describing its chance to be colonised by another patch:

$$
c_{i}^{\text {in }}=\frac{\beta}{(N-1) \mu} \sum_{j(\neq i)} A_{j}^{b} \exp \left(-\alpha d_{i j}\right)
$$

with the same parameters as in Eq. (2).

These equations (Eqs. 1-3) are appropriate for application to graph theory. We replace the 3 basic rates $\left(v_{i k}, C_{i}^{\text {out }}, C_{i}^{\text {in }}\right)$ of this model by quantities from the graph theory model of dispersal (see Appendix 1).

Consider a metapopulation consisting of discrete populations occupying $\mathrm{N}$ patches being described by a network $\mathrm{G}(V, E)$ with a finite set of vertices [ $V]$ connected by edges $[E]$ (for more on graph theory see Appendix 1). Each vertex $i$ has the attribute of area $\left(A_{i}\right)$ and each edge has an attribute of weight $\left(w_{i j}\right)$ indicating the strength of connection between 2 populations $\left(V_{i}, V_{j}\right)$ on vertex $i$ and vertex $j$, respectively.

The important point is that the weights $\left(w_{i j}\right)$, although describing the interaction (colonisation) between populations $\left(V_{i}, V_{j}\right)$, do not necessarily relate to area and distance of a patch. In the case of the James et al. (2002) study where the Lagrangian particle model provided the network edge weights (Kininmonth et al. 2010), the area of the patch approximately corresponds to the boundary length and this forms the basis for the number of particles released from each patch. Other dispersal models may use the entire patch area for the release and settlement of simulated larval particles. Crucially, the selected dispersal model only provides the weight of the connecting edges that link the non-dimensional vertices representing patches with area and quality values. Therefore, the 2 colonisation rates (Eqs. $2 \& 3$ ) have to be adapted to edge weights through summation of the inbound ( $w_{j i}$ from $j$ to $i$ ) and outbound ( $w_{i j}$, from $i$ to $j$ ) edge weights (from point of view of vertex $i$ ), respectively, for each vertex to measure the colonisation strength and colonisability as:

$$
\begin{aligned}
& C_{i}^{\text {out }}=\frac{S_{i}^{\text {out }}}{\mu}=\frac{1}{\mu} \sum_{j \in V(i)} W_{i j} \\
& C_{i}^{\text {in }}=\frac{s_{i}^{\text {in }}}{\mu}=\frac{1}{\mu} \sum_{j \in V(i)} W_{j i}
\end{aligned}
$$

where $v(i)$ is the set of neighbours of vertex $i$, and $s_{i}$ is the strength metric (see Eq. A1 in Appendix 1). For better understanding of the basic idea of Eqs. (4) \& (5), Fig. 1 demonstrates how $S_{i}$ can be calculated for a simple network of 3 vertices. The colonisation strength and colonisability of a patch, respectively, multiplied by the expected local population lifetime $(1 / v)$ of population $j$ (Eqs. $6 \& 7$ in Drechsler 2009) are then:

$$
\begin{aligned}
& u_{i}^{\text {in }}=\frac{1}{\mu}=\sum_{j \in V(i)} \frac{w_{j i}}{V_{j}} \\
& u_{i}^{\text {out }}=\frac{1}{\mu v_{i}}=\sum_{j \in V(i)} W_{i j}
\end{aligned}
$$

The harmonic mean $U_{i}$ of Eqs. (6) \& (7) (Eq. 8 in Drechsler 2009) then enters the aggregated colonisationextinction ratio for the entire network (Eq. 9 in Drechsler 2009), as:

$$
q=\prod_{i=1}^{N}\left(U_{i}\right)^{1 / N}
$$

Together with the geometric mean of the local extinction rates:

$$
V=\prod_{i=1}^{N} v_{i}^{1 / N}
$$

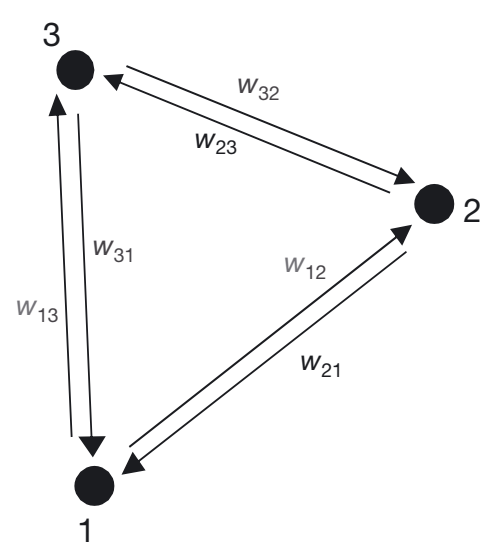

$$
\begin{aligned}
& s_{1}^{\text {out }}=w_{12}+w_{13} \\
& s_{2}^{\text {out }}=w_{23}+w_{21} \\
& s_{3}^{\text {out }}=w_{31}+w_{32} \\
& s_{1}^{\text {in }}=w_{21}+w_{31} \\
& s_{2}^{\text {in }}=w_{12}+w_{32} \\
& s_{3}^{\text {in }}=w_{13}+w_{23}
\end{aligned}
$$

Fig. 1. Determination of the sum $(s)$ of weights $(w)$ for a graph of 3 vertices $(1,2,3)$ 
the MLT of the metapopulation for the specified network configuration can be calculated by (Eq.11 in Drechsler 2009):

$$
\mathrm{MLT}=\frac{1}{V}=\sum_{i=k}^{N} \sum_{k=i}^{N} \frac{1}{k}\left(\frac{(N-i) !}{(N-k) !}\right) \frac{1}{(N-1)^{k-i}} q^{k-i}
$$

The decisive equations for the consideration of different topologies are Eqs. (4) \& (5), which relate metapopulation colonisation rates to quantities of the graph. Given these rates, the other equations follow the recipe of Drechsler (2009) to calculate MLT.

Model simulations. We firstly ask, for a dispersing larval pool of a set size, what effect does topology have on the MLT of the population? Secondly, what effect does the size of the metapopulation in relation to topology have on the MLT? Thirdly, how variable is the MLT given the distributions of edge weight and patch area that occur in the GBR?

We address the first question by examining the values of MLT for the configurations of Erdos-Renyi random, Tree, Lattice, Barabasi-Albert, Forest fire, Geometric (small and large radius), Complete and GBR small-world (Table 1, Fig. 2). One key parameter in the model is $\eta$, which describes the level of environmental stochasticity influencing the local extinction risk for a given patch area. Another key parameter is edge weight $\left(w_{i j}\right.$ and $\left.w_{j i}\right)$, which describes the strength of interaction (i.e. the number of dispersing individuals) between a patch $\left(V_{i}\right)$ and its neighbour $\left(V_{j}\right)$. Therefore, we study the impact of topography while stepping the total edge weight (sum of $w_{i j}$ and $w_{j i}$ for all patches) from 100 to 2500 in 50-unit increments. Following the
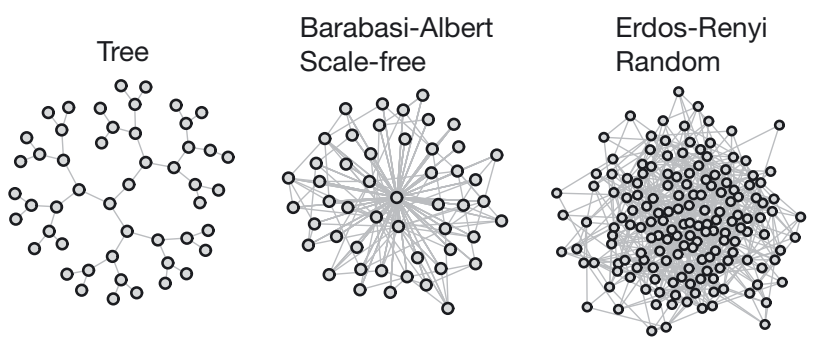

Geometric Small Radius

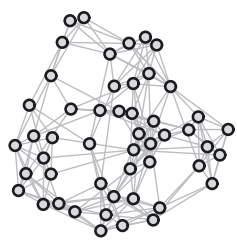

Geometric Large Radius

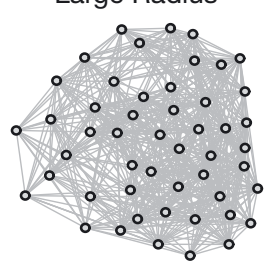

GBR small world

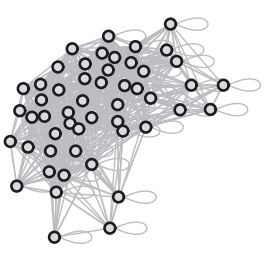

Forest Fire

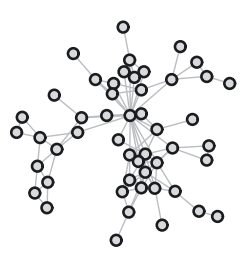

Lattice

Complete

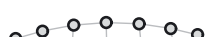
$\begin{array}{llllllll}0 & 0 & 0 & 0 & 0 & 0 & 0\end{array}$

00000000

00000000

000000000

00000000

00000000

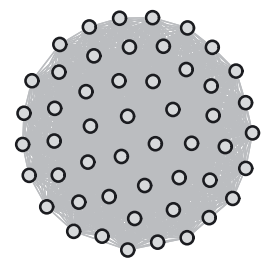

Fig. 2. Alternative topologies graph plots (size of 50 vertices) from Table 1

findings of Underwood et al. (2007) that the majority of Seriatopora hystrix larvae settle within $100 \mathrm{~m}$ of their natal colony, we set the area value at 2 ha. Exploration of MLT values for the $\eta$ and $\varepsilon$ parameter space indi-

Table 1. Alternative graphs for comparison (see Fig. 1)

\begin{tabular}{|c|c|}
\hline Model name & Description \\
\hline Erdos-Renyi random & $\begin{array}{l}\text { New vertices are added to the graph and } 4 \text { times the vertex number of new edges are } \\
\text { created connecting uniformly randomly chosen vertices (Erdos \& Renyi 1959). }\end{array}$ \\
\hline Complete & Every vertex is connected to every other node (Harary 1969). \\
\hline Forest fire & $\begin{array}{l}\text { Resembles how a forest fire spreads by igniting trees close by. Vertices are added } \\
\text { sequentially and edges are created with respect to the neighbouring configuration } \\
\text { (Leskovec et al. 2007). }\end{array}$ \\
\hline Barabasi-Albert scale free & $\begin{array}{l}\text { One vertex is added in each time step and edges are then created to link existing vertices } \\
\text { with a scale free probability, } P(k) \sim k^{-\gamma} \text { (Barabasi \& Albert 1999). }\end{array}$ \\
\hline Tree & $\begin{array}{l}\text { Based on a regular tree with } 2 \text { edges connecting the 'child' neighbours (Csardi \& } \\
\text { Nepusz 2006). The edges created are undirected. }\end{array}$ \\
\hline Lattice & $\begin{array}{l}\text { A lattice of } 2 \text { dimensions is composed of a set of } 4 \text { vertices joined in a square that is then } \\
\text { replicated for the specified size of the graph (Csardi \& Nepusz 2006). }\end{array}$ \\
\hline $\begin{array}{l}\text { Geometric } \\
\text { (small and large) }\end{array}$ & $\begin{array}{l}\text { First a number of points are dropped on to a unit square, then } 2 \text { points will be connected } \\
\text { with an undirected edge if they are closer to each other in Euclidean norm than a given } \\
\text { radius (Csardi \& Nepusz 2006). The small and large Geometric graphs used } 0.24 \text { and } \\
0.40 \text { radius values. }\end{array}$ \\
\hline GBR small-world & $\begin{array}{l}\text { Derived from a Lagrangian particle model within a wind and tide forced hydrodynamic } \\
\text { model. Graph constructed from the adjacency matrices (Kininmonth et al. 2010). }\end{array}$ \\
\hline
\end{tabular}


cated the parameter values with sufficient variation and acceptable ranges: $0.01<\eta<4,1<\varepsilon<100$. We then chose a rather small value of $\eta=0.1$, which represents weak environmental stochasticity and a large value of $\eta=2$, which represents strong environmental stochasticity (Drechsler 2009). The species-specific coefficient, $\varepsilon$, was set in the middle of the acceptable range, at 45 . The size of the network was 50 vertices, which is a compromise between processing time and network complexity.

The second question we address concerns network size, measured by the number of vertices, $\mathrm{N}$. The networks (Erdos-Renyi random, Tree, Lattice, BarabasiAlbert, Forest fire, Geometric [small and large radius], Complete and GBR small-world; Table 1, Fig. 2) were created from $N=10$ and increased to 155 by 5 -unit increments. The total edge weight for each network was set at 1500 , the extinction-area exponent, $\eta$, varied from 0.1 to $2, \varepsilon$ was set at 45 and the area for each vertex was set at 2 ha. Since the size of the population also relates to the area of the patch, we vary the area uniformly in a subsequent simulation from 0.1 to 3 ha. Total edge weight was set at $1500, \eta$ varied from 0.1 to 2 , and $\varepsilon$ was set at 45 (scaled to area units).

The third question was examined by sampling the GBR network (Kininmonth et al. 2010) from 10 to 160 vertices in multiples of 50 while recording the value of MLT. The patch area and edge weights are directly obtained from the GBR network. The network used contained 321 vertices and 7487 edges and was modelled for 1998 with parameters of day release, $1 \mathrm{~km}$ pickup zone, $1 \mathrm{wk}$ pre-competence, $3 \mathrm{wk}$ competent and no predation (James et al. 2002).

The simulations were run in R with primary use of igraph package (Csardi \& Nepusz 2006).

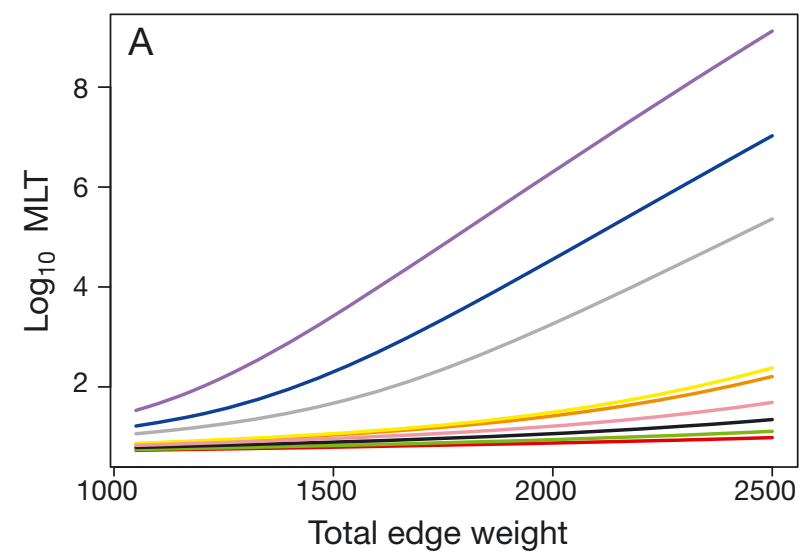

\section{RESULTS}

Increasing edge weight caused MLT values to increase exponentially (Fig. 3).Topologies such as the Geometric and Tree increased strongly with an increase in total edge weight, whereas other topologies such as Lattice and Complete increased only gradually. The effect of increasing edge weight was to increase the interaction strength between vertices and thus accentuate the topologies determining the distribution of connections. Topologies such as the Geometric and Forest fire (Fig. 2) can rapidly compact the network since the vertices within the hubs have a disproportionately higher number of edges (compared to the majority of vertices outside of hubs) that connect to other vertices that also have a higher number of edges. Consequently, small increases in edge weight will bind the vertices in the hubs closer together. The Geometric topology is similar to the topology considered in the distance dispersal models used by Drechsler (2009) and Frank \& Wissel (2002). It was ranked 1 and 3 in the 2 sets of simulations with varying $\eta$ from 0.1 to 2 . Topologies with evenly distributed degrees, such as the Lattice, only compact the local neighbourhood as the edge weight increases. The Complete topology increased the least, and this is due to the even division of the total edge weight by the maximum number of edges resulting in relatively 'weak' edges that respond slowly to weight increases. Apart from Tree, Lattice and Complete, networks were constructed in a stochastic manner and the MLT values varied slightly with each network simulation.

When network size was increased, MLT increased at least exponentially for all topologies (Fig. 4). The Geometric, Forest fire and GBR small-world topologies increased at the highest rates while the Lattice and

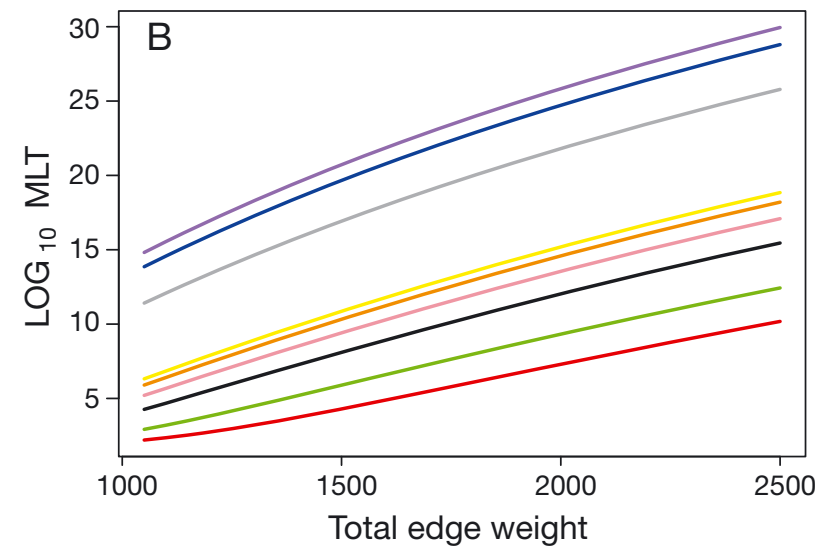

Fig. 3. Effect of topology on the mean life time (MLT) with varying total edge weights and extinction-area parameter: $(A) \eta=0.1$, (B) $\eta=2$. Topologies, with highest MLT values first: Geometric small (purple), Tree (blue), Geometric large (grey), Erdos-Renyi random (yellow), Forest fire (orange), Great Barrier Reef (GBR) small-world (pink), Barabasi-Albert (black), Lattice (green), Complete (red) 

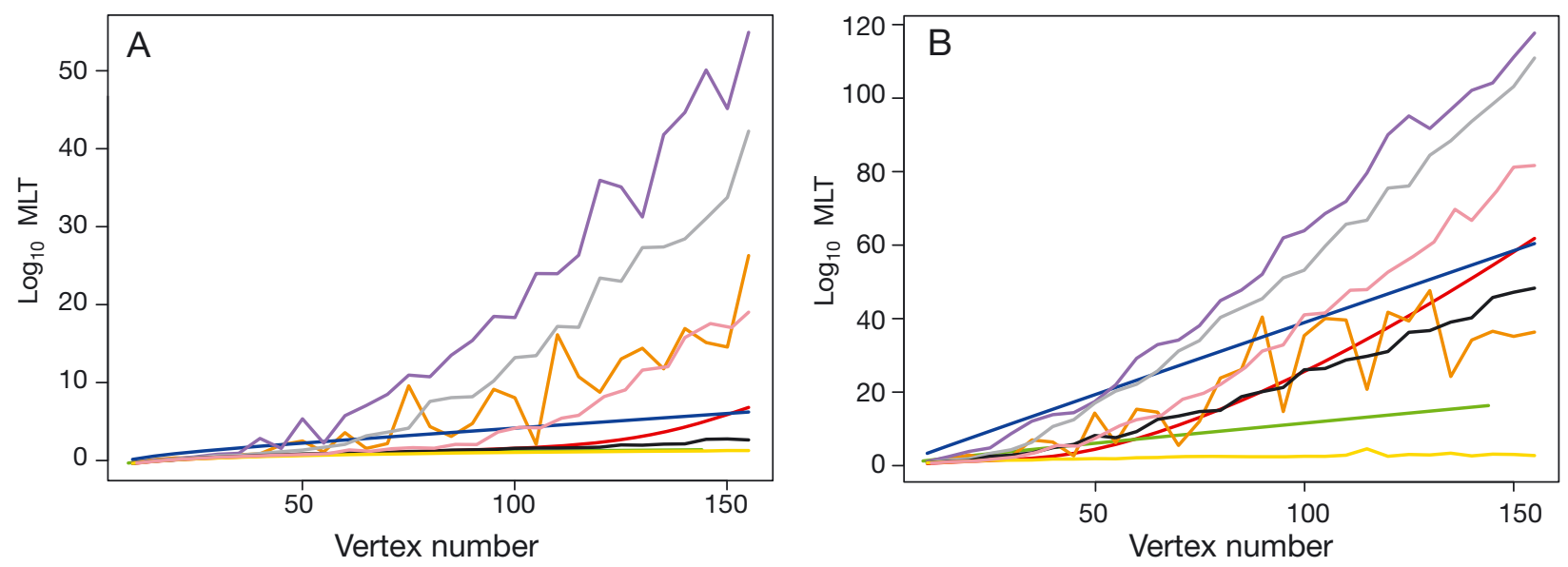

Fig. 4. Change in mean life time (MLT) with increasing number of vertices and corresponding increase in total weight of the dispersal network. Extinction-area parameter (A) $\eta=0.1$, (B) $\eta=2$. Topologies, with highest MLT values first: Geometric small (purple), Geometric large (grey), Forest fire (orange), GBR small-world (pink), Tree (blue), Complete (red), Barabasi-Albert (black), Lattice (green), Erdos-Renyi random (yellow). Variability for some topologies is due to their stochastic generation creating different numbers of edges

Erdos-Renyi random topologies increased much more slowly. From a metapopulation perspective, the topologies with tightly bound clusters ('communities' in this context) were quickly forming highly persistent 'hubs' that served to enhance dispersal.

In the vertex number simulation above, each vertex added to the network also added additional area. To isolate the effects of area changes, we simulated the increase in area ( 0.1 to 3.0 ha by 0.1 increments) but kept the vertex number at 50 . For a high extinction rate $(\eta=$ 0.1 ), MLT increased logarithmically with respect to increased area (Fig. 5A). For a lower $\eta(=2)$, there was an initial gradual increase for the area range of 0.1 to 1 for all topologies, followed by an exponential change in
MLT but at different area values for each topology type (Fig. 5B). The Geometric topology ranked the highest with respect to MLT, whereas the Complete topology was lowest. Similar to the previous simulations, networks with a small number of highly connected clusters generated higher MLT values as the area was increased.

The GBR small-world network is a heterogeneous mix of patch (coral reef) areas, dispersal strengths (derived from a Lagrangian particle dispersal model) and topologies (Fig. 6). In our simulations, as the size of the sub-sampled network increased from 10 to 160 vertices, mean MLT increased logarithmically (Fig. 7A). Standardising for the change in mean MLT values, MLT variation decreased as expected (Fig. 7B).

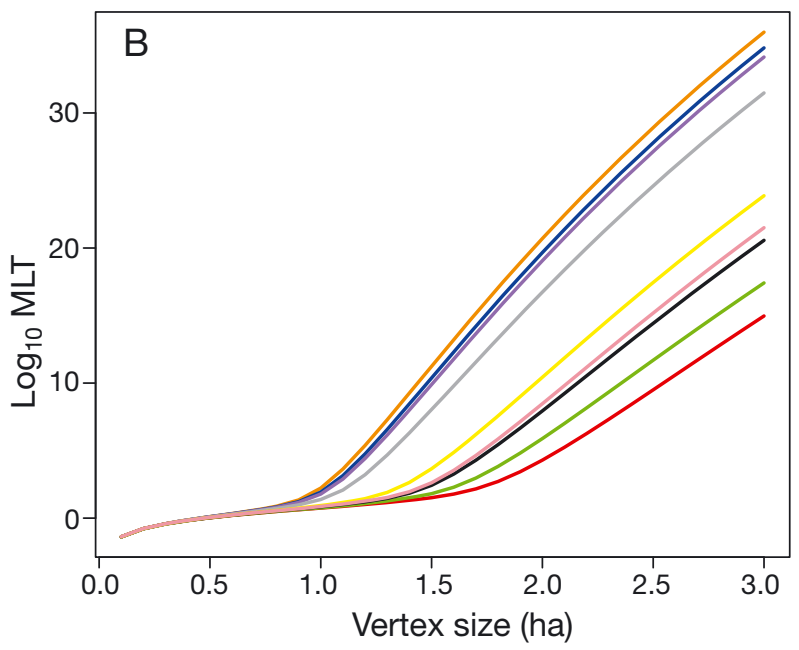

Fig. 5. Mean life time (MLT) as area of each patch is increased from 0.1 to 3 ha, with extinction-area parameter (A) $\eta=0.1$ and (B) $\eta=2$. Topologies, with highest MLT values first: Geometric small (purple), Tree (blue), Geometric large (grey), Forest fire (orange), Erdos-Renyi random (yellow), GBR small-world (pink), Barabasi-Albert (black), Lattice (green), Complete (red) 


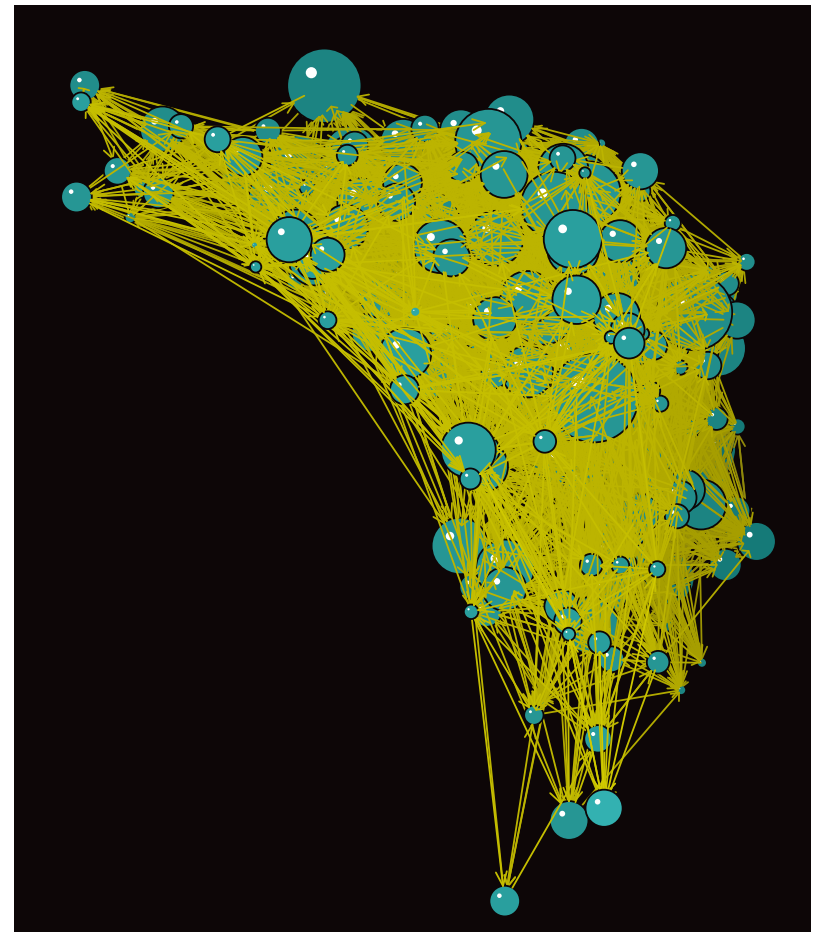

Fig. 6. Great Barrier Reef small-world network (for year 1998) where the size of each vertex (ball) is in proportion to the harmonic mean ( $U_{i}$ Eq. 9). Image created using the Fruchterman-Reingold algorithm in Pajek (Batagelj \& Mrvar 1998) and KiNG (http://kinemage.biochem.duke.edu) using network modelled for year 1998 with parameters of day release, $1 \mathrm{~km}$ pickup zone, $1 \mathrm{wk}$ pre-competence, $3 \mathrm{wk}$ competent and no predation (James et al. 2002)

\section{DISCUSSION}

The capacity of metapopulation modelling to inform conservation planning is based on the assumption that dominant metapopulation processes are represented in the model (Mumby 1999). For dispersal, this has been limited in the past to simple distance-dependent dispersal kernels, assuming a homogenous landscape matrix and non-directional organism movement. This is adequate when the simple homogeneous nature of the landscape matrix does not impair the passage of propagules, but in marine ecosystems, the dynamic nature of the transport processes results in a significant and varying proportion of propagules arriving at distant habitats (Kinlan \& Gaines 2003). This creates dispersal patterns that are complex and best represented by networks (Bodin \& Norberg 2007, Bascompte 2009). Metapopulation modelling based on these networks is likely to capture the topological structures relevant to conservation planning.

We modified the MLT metapopulation model of Drechsler (2009) so that the parameters could be directly referred to graph theory metrics. Eqs. (1) to (10) presented here can be used to evaluate conservation planning scenarios with respect to the topology of networks. Since estimation of the parameters in the field is difficult, the main benefit of using this model is the comparative metapopulation response to differing conservation alternatives (e.g. distribution of marine reserves).

The structural configuration of networks (= topology) influenced MLT. Simulated networks with strongly
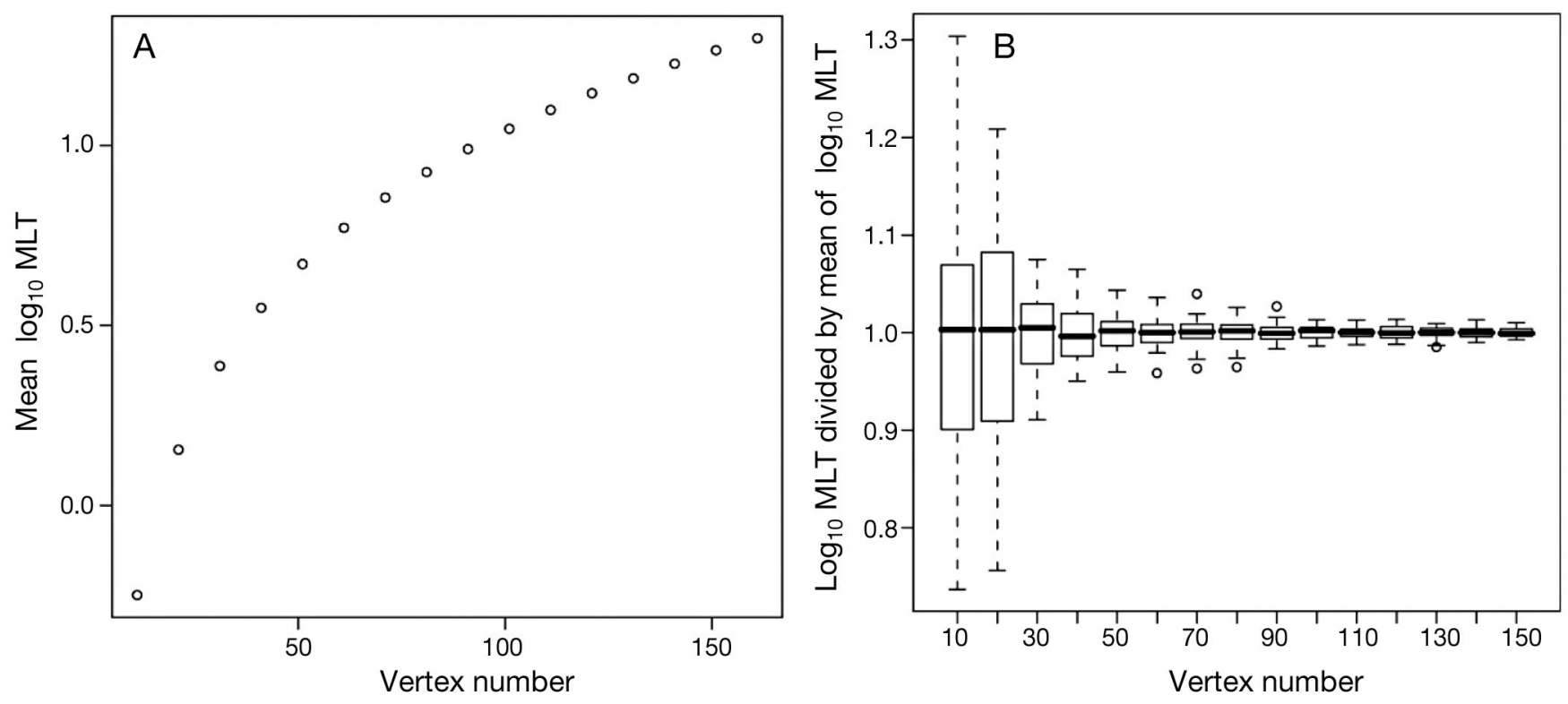

Fig. 7. (A) Increasing mean $\log _{10}$ MLT for the Great Barrier Reef (GBR) network subsamples at size range $10-150$ vertices with 50 repetitions. (B) $\log _{10}$ MLT values standardised by mean $\log _{10}$ MLT across the network size range showing decreasing variance with network size. MLT: mean life time 
clustered topologies (standardized with uniform patch area and edge weights), such as a Geometric network, usually had longer MLT values than networks with evenly distributed linkages such as a Lattice. This implies that preserving core 'hubs' (sensu Li et al. 2005) where a small set of strongly interconnected vertices exists will increase the MLT of a metapopulation.

Two key determinants of the MLT were the extinction rate and the colonisation rate. Colonisation rate was a function of the weight value of the edges. When the total edge weight value for the network was increased, the network effectively contracted (from a larval dispersal perspective) and MLT increased. This highlights the positive effect that increases in edge weight, inferring connectedness, has on the stability of local populations. Conversely, this model supports the paradigm that isolating populations will dramatically increase the risk of local extinction (Hanski 1998). Since the distribution of connections and edge weights in nature is determined by environmental conditions and will likely be heterogeneous, individual populations will have variable colonisation-extinction ratios.

Similarly, extinction risk is likely to vary across metapopulations as environmental conditions vary, and lead to variation in habitat quality for different populations, sometimes producing source-sink dynamics. Populations in high quality (low extinction risk) habitats are able to release larvae into the dispersal network and thus act as sources (Crowder et al. 2000). Conversely, poor quality (high extinction risk) habitats will absorb the migrant larvae but will have limited capacity to contribute to the regional population larval source (Crowder et al. 2000). The simulations conducted here used a homogeneous extinction measure, but the MLT model design presented here can easily accommodate variation in habitat quality.

This model shows that as network size increases, MLT varies by network topology. Topologies with a smallworld configuration generate networks with a higher MLT compared to those with homogenous degree distributions. Simply increasing the number of populations in a metapopulation with a Lattice or Erdos-Renyi random topology does little to increase MLT. The mean field approach within this model will only have an effect on the MLT when there is a shift in the distribution of the colonisation strength and colonisability values. Homogeneous topologies can increase in size, but the geometric mean of their aggregated colonisation-extinction ratio remains constant. Increases in patch number most effectively increase MLT if at the same time the geometric mean of the colonisation-extinction ratios is increased. If patch sizes are kept constant, changes in network topology are necessary to alter MLT.

Populations that occupy a small geographic range or occur in small distributed populations are considered vulnerable to extinction (Hanski 1998). This model provides further support that maximising metapopulation effective size (i.e. increased number of patches that can connect to the network) through the reduction of fragmentation or the protection of critical habitat is an effective conservation activity. Increasing the area of each of the patches also increased MLT but with a different effect than simply adding more patches to the network. Addition of high quality patch area, with a corresponding lower extinction rate, caused large increases in MLT. Thus, extinction rate significantly influences the effect that extra vertices have on MLT. This implies that adding 'poor quality' patches to a metapopulation has minimal advantage unless there exists 'hubs' of strongly connected patches.

The implication for conservation planners from these 2 scenarios (increase area, increase patch number) is that the first stage of a recovery plan should be to protect the existing area and ideally the area constituting hubs, if they exist (Vuilleumier \& Fontanillas 2007). Subsequent effort should be allocated to increasing the number of effective populations, perhaps by enhancing connectivity to isolated patches (highest priority to the high quality patches). Of course this assumes that environmental catastrophes can be mitigated; otherwise, it might be prudent to spread conservation efforts in space (Game et al. 2008).

Many naturally occurring networks exhibit a smallworld topology (Green \& Sadedin 2005), which ranked highly with respect to MLT in our simulations. The small-world topology was represented in differing forms by the Geometric, Forest fire and GBR networks. Each of these generated networks that contained clusters of highly connected vertices (hubs) that were also connected to each other. From a hierarchical perspective, the vertices in these hubs can be aggregated to simplify the network into a connected series of hubs (Ravasz \& Barabasi 2003, Lancichinetti et al. 2009). The topological effect on larval dispersal is to optimise local interchange, with some dispersal occurring, at high cost, between distant populations. By placing the area available to generate propagules and also receive migrants in close proximity, the aggregated colonisationextinction ratio was maximised. Topologies that generate networks with evenly distributed connections, such as the Complete, Lattice and Erdos-Renyi random networks, will limit the concentration of migration and immigration potential across the network.

As the weight or area of vertices increased, the small-world networks formed consolidated clusters quicker than other topologies. Likewise, as the number of vertices was increased, the effective size of a smallworld network remained small (Grossman 2000). The Lattice configuration, however, expanded proportionally to the number of vertices (and effectively reduced 
propagule density) as newer vertices were added. For a small-world even when we used the scale free distribution of edge weight generated by the Lagrangian particle model (Kininmonth et al. 2010), the distribution of the colonisation-extinction ratio remained spread across the network.

Random sampling of the GBR small-world network yielded a diversity of topologies. Small networks extracted from the large Complete network showed high variability, but as sample size was increased to include half the GBR small-world network, variability decreased. This emphasises the dilemma for the conservation planner regarding local versus regional protection plans for a complex system (Drechsler \& Wissel 1998). Can custom-designed local plans be integrated into a holistic regional plan? In particular, for the GBR can isolated coastal coral reefs with a Tree-like dispersal topology be protected in the same way as the more numerous mid-shelf reefs, which have a Geometric dispersal topology? This model suggests that where local populations have a dispersal topology that is homogeneous (i.e. Lattice or Complete), the conservation focus should remain on identifying and enhancing habitat quality (Almany et al. 2009). Otherwise this model suggests that the priority should be to protect the quality of the 'source' patches especially if they have strongly weighted or numerous connections to neighbouring patches (i.e. part of a hub).

\section{Application to GBR conservation}

The spatial allocation of marine reserves in the GBR in 2004 was conducted with knowledge of the biophysical regime (Fernandes et al. 2009). One of these principles was to maximise the use of environmental information to determine the configuration of no-take areas to form viable networks. However, the requisite data was inadequate and the theoretical framework for accommodating the spatial configuration was lacking (Fernandes et al. 2009). As a consequence, the reserve allocation process was primarily focused on the capture of representative biodiversity within a reserve system (Kerrigan et al. 2010) without attention to metapopulation processes. The primary management tool in the GBR reserve system is the reduction of fishing pressure (equivalent to the reduction of the extinction rate) for sessile adults. The spatial allocation of reduced extinction risk that maximizes MLT of marine populations can also form the basis for a comprehensive conservation framework. The GBR exhibits a range of larval dispersal topologies at differing scales (Kininmonth et al. 2010), so optimising the colonisation processes (through the enhancement of habitat quality via a reduction in extinction rate) at local and regional scales will be dependent on the topological patterns. The algorithms to formulate this design process are the topic of ongoing research.

This new method of integrating the MLT formulae within the network structure presents significant enhancements over the previous spatial metapopulation models. In particular, the capacity to identify topological influences on MLT, where the dispersal processes are not operating in a homogeneous environment, is an important advance. As dispersal networks describing various ecological systems are developed, the capacity to understand the complex interactions between network size, patch area, environmental stochasticity and topology will surge. Consequently, effective protection measures with respect to topology will foster implementation of optimal conservation efforts for marine metapopulations.

Acknowledgements. We thank the Marine Modelling Unit of James Cook University for the provision of dispersal model outputs for the GBR. The comments of the supporting editor R. N. Lipcius and 3 anonymous reviewers were very helpful in the revision of the manuscript.

\section{LITERATURE CITED}

Albert R, Barabasi AL (2002) Statistical mechanics of complex networks. Rev Mod Phys 74:47-94

Almany GR, Connolly S, Heath DD, Hogan JD and others (2009) Connectivity, biodiversity conservation and the design of marine reserve networks for coral reefs. Coral Reefs 28:339-351

Barabasi AL, Albert R (1999) Emergence of scaling in random networks. Science 286:509-512

Barabasi A-L (2002) Linked: the new science of networks, Vol 1. Perseus Publishing, Cambridge, MA

Bascompte J (2009) Disentangling the web of life. Science 325:416-419

Bascompte J, Sole R (1996) Habitat fragmentation and extinction thresholds in spatially explicit models. J Anim Ecol 65: 465-473

Batagelj V, Mrvar A (1998) Pajek: program for large network analysis. Ljubljana. Connections 21:47-57. http://pajek. imfm.si/doku.php?id=pajek

Bjornstad ON, Ims R, Lambin X (1999) Spatial population dynamics: analyzing patterns and processes of population synchrony. Trends Ecol Evol 14:427-432

Bode M, Bode L, Armsworth PR (2006) Larval dispersal reveals regional sources and sinks in the Great Barrier Reef. Mar Ecol Prog Ser 308:17-25

> Bode M, Burrage K, Possingham HP (2008) Using complex network metrics to predict the persistence of metapopulations with asymmetric connectivity patterns. Ecol Modell 214:201-209

Bodin O, Norberg J (2007) A network approach for analyzing spatially structured populations in fragmented landscape. Landscape Ecol 22:31-44

Colizza V, Pastor-Satorras R, Vespignani A (2007) Reactiondiffusion processes and metapopulation models in heterogeneous networks. Nat Phys 3:276-282

Crowder LB, Figueira WF (2006) Metapopulation ecology and marine conservation. In: Kritzer JP, Sale PF (eds) Marine metapopulations. Elsevier, New York, NY 
Crowder LB, Lyman SJ, Figueira WF, Priddy J (2000) Sourcesink population dynamics and the problem of siting marine reserves. Bull Mar Sci 66:799-820

Csardi G, Nepusz T (2006) The igraph software package for complex network research. Interjournal Complex Systems, available at: http://necsi.org/events/iccs6/view abstract.php?id=88

Dorogovtsev SN, Mendes J (2002) Evolution of networks. Adv Phys 51:1079-1187

Drechsler M (2009) Predicting metapopulation lifetime from macroscopic network properties. Math Biosci 218:59-71

> Drechsler M, Wissel C (1998) Trade-offs between local and regional scale management of metapopulations. Biol Conserv 83:31-41

Erdos P, Renyi A (1959) On random graphs. Publicationes Mathematicae 6:290-297

Erdos P, Renyi A (1960) On the evolution of random graphs. Publ Math Inst Hung Acad Sci 5:17-60

> Fernandes L, Day JR, Kerrigan B, Breen D and others (2009) A process to design a network of marine no-take areas: lessons from the Great Barrier Reef. Ocean Coast Manage 52:439-447

- Franc A (2004) Metapopulation dynamics as a contact process on a graph. Ecol Complex 1:49-63

> Frank K, Wissel C (1998) Spatial aspects of metapopulation survival: from model results to rules of thumb for landscape management. Landscape Ecol 13:363-379

Frank K, Wissel C (2002) A formulae for the mean lifetime of metapopulations in heterogeneous landscapes. Am Nat 159:530-552

Game ET, Watts ME, Wooldridge S, Possingham H (2008) Planning for persistence in marine reserves: a question of catastrophic importance. Ecol Appl 18:670-680

Gerber LR, Botsford LW, Hastings A, Possingham HP, Gaines SD, Palumbi SR, Andelman S (2003) Population models for marine reserve design: a retrospective and prospective synthesis. Ecol Appl 13:S47-S64

Graham EM, Baird AH, Connolly S (2008) Survival dynamics of scleratinian coral larvae and implications for dispersal. Coral Reefs 27:529-539

> Green DG, Sadedin S (2005) Interactions matter: complexity in landscapes and ecosystems. Ecol Complex 2:117-130

Gross J, Yellen J (1999) Graph theory and its applications, Vol 1. CRC Press, Boca Raton, FL

> Grossman J (2000) Review of small worlds: the dynamics of networks between order and randomness. Am Math Mon 107:664-668

- Hanski I (1998) Metapopulation dynamics. Nature 396:41-49

Harary F (1969) Graph theory, Vol 1. Addison-Wesley, Reading, MA

Hughes T, Baird AH, Dinsdale EA, Moltschaniwskyj NA, Pratchett MS, Tanner JE, Willis BL (1999) Patterns of recruitment and abundance of corals along the Great Barrier Reef. Nature 397:59-63

> James MK, Armsworth P, Mason L, Bode L (2002) The structure of reef fish metapopulations: modelling larval dispersal and retention patterns. Proc Biol Sci 269: 2079-2086

Jeong H, Tombar B, Albert R, Oltvai Z, Barabasi AL (2000) The large-scale organization of metabolic pathways. Nature 407:651-654

Jones GP, Planes S, Thorrold SR (2005) Coral reef fish larvae settle close to home. Curr Biol 15:1314-1318

Junker B, Schreiber F (2008) Analysis of biological networks. Wiley-Interscience, Hoboken, NJ

Kaplan DM (2006) Alongshore advection and marine reserves: consequences for modeling and management. Mar Ecol Prog Ser 309:11-24
Kerrigan B, Breen D, De'ath G, Day JR, Fernandes L, Tobin R, Dobbs K (2010) Classifying the biodiversity of the Great Barrier Reef World Heritage Area. Great Barrier Marine Park Authority, no. 104, Townsville

Kingsford M, Leis J, Shanks AL, Lindeman $\mathrm{K}$, Morgan $\mathrm{S}$, Pineda J (2002) Sensory environments, larval abilities and local self-recruitment. Bull Mar Sci 70:309-340

Kininmonth S, De'ath G, Possingham HP (2010) Graph theoretic topology of the Great but small Barrier Reef world. Theor Ecol 3:75-88

> Kinlan BP, Gaines SD (2003) Propagule dispersal in marine and terrestrial environments: a community perspective. Ecology 84:2007-2020

Kritzer J, Sale P (2004) Metapopulation ecology in the sea: from Levins' model to marine ecology and fisheries science. Fish Fish 5:131-140

Lancichinetti A, Fortunato S, Kertesz J (2009) Detecting the overlapping and hierarchical community structure in complex networks. N J Phys 11:033015

> Leskovec J, Kleinberg J, Faloutsos C (2007) Graph evolution: densification and shrinking diameters. ACM Transactions on Knowledge Discovery from Data 1(1): Article 2

Levins R (1969) Some demographic and genetic consequences of environmental heterogeneity for biological control. Bull Entomol Soc Am 15:237-240

Li L, Alderson D, Doyle JC, Willinger W (2005) Towards a theory of scale-free graphs: definition, properties and implications. Internet Math 2:431-523

Lipcius RN, Stockhausen WT, Eggleston DB (2001) Marine reserves for Caribbean spiny lobster: empirical evaluation and theoretical metapopulation recruitment dynamics. Mar Freshw Res 52:1589-1598

Lipcius RN, Eggleston DB, Schreiber SJ, Seitz RD and others (2008) Importance of metapopulation connectivity to restocking and restoration of marine species. Rev Fish Sci 16:101-110

Moilanen A, Wilson KA, Possingham H (eds) (2009) Spatial conservation prioritization. Oxford University Press, London

Montoya J, Sole R (2002) Small World patterns in food webs. J Theor Biol 214:405-412

Mumby P (1999) Can Caribbean coral populations be modelled at metapopulation scales? Mar Ecol Prog Ser 180: $275-288$

> Newman ME (2004) Analysis of weighted networks. Phys Rev E Stat Nonlin Soft Matter Phys 70:056131

> Nicholson E, Possingham H (2007) Making conservation decisions under uncertainty for the persistence of multiple species. Ecol Appl 17:251-265

Oliver J, Willis BL (1987) Coral-spawn slicks in the Great Barrier Reef: preliminary observations. Mar Biol 94:521-529

> Ovaskainen O, Hanski I (2001) Spatially structured metapopulation models: global and local assessment of metapopulation capacity. Theor Popul Biol 60:281-302

Ovaskainen O, Sato K, Bascompte J, Hanski I (2002) Metapopulation models for extinction threshold in spatially correlated landscapes. J Theor Biol 215:95-108

Proulx S, Promislow D, Phillips P (2005) Network thinking in ecology and evolution. Trends Ecol Evol 20:345-353

Pulliam HR (1988) Sources, sinks and population regulation. Am Nat 132:652-661

- Ravasz E, Barabasi A-L (2003) Hierachical organisation in complex networks. Phys Rev E Stat Nonlin Soft Matter Phys 67:026112

Rozenfeld AF, Arnaud-Haond S, Hernandez-Garcia E, Egiluz V, Serrao E, Duarte C (2008) Network analysis identifies weak and strong links in a metapopulation system. Proc 
Natl Acad Sci USA 105:18824-18829

Sale PF, Hanski I, Kritzer JP (2006) The merging of metapopulation theory and marine ecology: establishing the historical context. In: Kritzer JP, Sale PF (eds) Marine Metapopulations. Elsevier, New York, NY

Schnettler S (2009) A structured overview of 50 years of small-world research. Soc Networks 31:165-178

Steuer R, Lopez G (2008) Global network properties. In: Junker B, Schreiber F (eds) Analysis of biological networks. Wiley-Interscience, Hoboken, NJ

Treml E, Halpin PN, Urban D, Pratson L (2008) Modelling population connectivity by ocean currents: a graphtheoretic approach for marine conservation. Landscape Ecol 23:19-36

Underwood JN, Smith LD, Van Oppen M, Gilmour J (2007) Multiple scales of genetic connectivity in a brooding coral on isolated reefs following catastrophic bleaching. Mol Ecol 16:771-784

Underwood JN, Smith LD, Van Oppen M, Gilmour J (2009) Ecologically relevant dispersal of corals on isolated reefs: implications for managing resilience. Ecol Appl 19:18-29

Urban D, Keitt T (2001) Landscape connectivity: a graphtheoretic perspective. Ecology 82:1205-1218
Urban D, Minor E, Treml E, Schick RS (2009) Graph models of habitat mosaics. Ecol Lett 12:260-273

van Oppen M, Lutz A, De'ath G, Peplow L, Kininmonth S (2008) Genetic traces of recent long-distance dispersal in a predominantly self-recruiting coral. PLoS ONE 3(10):e3401

Vuilleumier S, Fontanillas P (2007) Landscape structure affects dispersal in the greater white-toothed shrew: inference between genetic and simulated ecological distances. Ecol Modell 201:369-376

Vuilleumier S, Metzger R (2006) Animal dispersal modelling: handling landscape features and related animal choices. Ecol Modell 190:159-170

Vuilleumier S, Wilcox C, Cairns B, Possingham HP (2007) How patch configuration affects the impact of disturbances on metapopulation persistence. Theor Popul Biol 72:77-85

Watts D, Strogatz S (1998) Collective dynamics of 'smallworld' networks. Nature 393:440-442

Wilcox C, Possingham H (2002) Do life history traits affect the accuracy of diffusion approximation for mean time to extinction? Ecol Appl 12:1163-1179

Wilkinson CR (2000) Status of coral reefs of the World: 2002. Australian Institute of Marine Science, Townsville

\section{Appendix 1. Graph theory}

Graph theory is a branch of mathematics that describes the nature of simple (Harary 1969, Gross \& Yellen 1999) and complex (Dorogovtsev \& Mendes 2002, Proulx et al. 2005, Steuer \& Lopez 2008) networks. A graph $G(V, E)$ can be defined as a finite set of vertices $[V]$ connected by edges $[E]$ (Fig. A1). Theorems of the properties of simple graphs dominated the use of graph theory until the advent of fast computers. Large graphs were modelled as randomly assembled graphs or as regular lattices (Albert \& Barabasi 2002).

The degree $\left(k_{i}\right)$ of a vertex $i$ defines the number of connecting edges and hence the number of neighbouring vertices (Fig. A1). All vertices in a regular lattice share a common degree, $\langle k\rangle$, and this configuration (often displayed as a grid of equally sized cells) is commonly used as the basis for neighbourhood interaction models (Bjornstad et al. 1999, Vuilleumier \& Metzger 2006). In a random graph, the degree of all the vertices has a Poisson distribution and is characterised by a modal hump at the mean degree (Erdos \& Renyi 1960, Albert \& Barabasi 2002). The average degree for all the vertices in a graph is denoted as: $\langle k\rangle$.

Fig. A1. Simple graph containing 5 vertices and 7 edges. Four of the vertices have a degree of 3 , one has a degree of 2

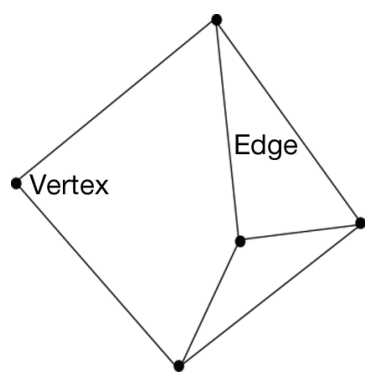

Graphs that have a high level of clustering (triangular linkages) and average short path lengths (number of edges from one vertex across the network to another vertex) compared with random or regular lattices are known as 'smallworld' graphs (Watts \& Strogatz 1998). However, there is no explicit definition of the properties of small-world graphs (Schnettler 2009). Small-world topology increases the connectivity of vertices, effectively making it smaller, in terms of interactions, than a random or regular one. Clusters connected by longer links enable any point in the network to be located a small number of steps from any other point, and this forms the basis of Stanley Milgram's 6 degrees of separation for the network describing human social interactions (Barabasi 2002).

While the number of connections reveals many of the topological structures, the strength of each connection can be used to describe the exchanges between the vertices. In the case where the strength of connection between 2 vertices is known, the value of the edge weight $\left(w_{i j}\right)$ describes the intensity of interaction occurring between vertex $i$ to vertex $j$. The inbound and outgoing strength indices for vertex $i$ are defined (Newman 2004) as:

$$
S_{i}^{\text {in }}=\sum_{j \in V(i), j \in E_{j i}} w_{j i}, \quad s_{i}^{\text {out }}=\sum_{j \in V(i), j \in E_{i j}} w_{i j}
$$

where $v(i)$ is the set of neighbours of vertex $i, E_{j i}$ is the set of edges having the inbound direction to vertex $i$ and $E_{i j}$ is the complementary set of edges having an outbound origin from vertex $i$. The average inbound and outbound strengths for the entire graph are:

$$
s^{\text {in }}=\frac{1}{n} \sum_{i=1}^{n} s_{i}^{\text {in }} \quad \text { and } \quad s^{\text {out }}=\frac{1}{n} \sum_{i=1}^{n} s_{i}^{\text {out }}
$$

